\title{
LA APROPIACIÓN SOCIAL DE LA CIENCIA, LA TECNOLOGÍA Y LA INNOVACIÓN Y LOS ORGANISMOS DE COOPERACIÓN INTERNACIONAL.
}

\author{
THE SOCIAL EMPOWERMENT OF SCIENCE, TECHNOLOGY, \\ AND INNOVATION AND THE INTERNATIONAL COOPERATION AGENCIES.
}

Recibido: marzo de 2012 - Revisado: junio de 2012 - Aceptado: 30 de noviembre de 2012

Por: Ibelis Blanco Rangel ${ }^{1}$.

\section{RESUMEN:}

La ciencia, la tecnología y la innovación son elementos claves para el desarrollo económico y social de un país, sin embargo no son tema de amplia discusión desde la cooperación internacional para el desarrollo, es por ello, teniendo presente la importancia que ha cobrado la transmisión y masificación de ese conocimiento propuesta por Colciencias a través de la apropiación social de la Ciencia, la Tecnología y la Innovación, interesa conocer cuáles han sido los aportes de las instituciones de cooperación internacional en América latina en general y cuales los posibles retos para Colombia.

\section{PALABRAS CLAVES:}

ciencia, tecnología, innovación, apropiación social, cooperación internacional.

\section{ABSTRACT:}

Science, technology, and innovation are key elements for economic and social development in a country; however they are not a wide discussion topic from international cooperation for development. That is why, bearing in mind the importance that the transmission and the massification of that knowledge have gained, and which is proposed by Colciencias through the social empowerment of science, technology, and innovation; it is interesting to know which contributions the institutions of international cooperation have had in Latin America, in broad terms, as well as the general potential challenges that Colombia has to face.

\section{KEY WORDS AND EXPRESSIONS:}

Science, technology, innovation, social empowerment, and international cooperation.

\footnotetext{
${ }^{1}$ Ibelis C. Blanco Rangel. Socióloga de la Universidad Católica Andrés Bello de Venezuela, cursó estudios de postgrado en Gerencia de proyectos de investigación y desarrollo en la Universidad Central de Venezuela, Especialista en Cooperación Internacional para el Desarrollo y de la Universidad de San Buenaventura seccional Cartagena_Colombia, en convenio con la Universidad de Pavia, Italia. Ha desarrollado su experiencia profesional en investigación social y evaluación de proyectos. Actualmente, se desempeña como investigadora de la Escuela Latinoamericana de Cooperación y Desarrollo de la Universidad de San Buenaventura y es candidata a Magister en Cooperación Internacional. ibelis.blanco@gmail.com
} 


\section{Institucionalización de la ciencia la tecnologia y la innovación en América Latina.}

"el mundo del conocimiento se mueve en la lógica de la racionalidad científica, la cual ya no puede estar solamente en las cabezas de los científicos sino que debe ser patrimonio común de la gente”. (Ahumada \& Miranda. 2003).

A partir de las consecuencias que desencadenaron luego de la segunda guerra mundial, la ciencia y la tecnología cobraron un papel importante en el escenario global, al finalizar a ello se le sumó la interdependencia económica entre los países. Además de la ciencia y la tecnología, la innovación, fue considerada un punto claves de para el desarrollo de las naciones, en especial para transitar a la sociedad del conocimiento, por ello, es necesario identificar cuales organismos de cooperación internacional que han participado en el proceso de institucionalización el tema y en América Latina.

Desde 1948 se inició en Latinoamérica el proceso de formulación y diseño de políticas en materia de ciencia y tecnología, en ese año se reunieron en Montevideo un grupo de investigadores cuyo resultado fue la creación del Centro de Cooperación Científica en América Latina. (Lemarchand, 2010).

Posteriormente, en 1960 se realizó en Caracas el Seminario Regional sobre la Organización de la Investigación Científica en Latinoamérica, de la cual derivó la declaración de Caracas. En ella se plantearon consideraciones que hoy dia siguen siendo vigentes, es decir, metas por alcanzar, tal es el caso de la importancia de: el papel de la ciencia y la tecnología en la opinión pública; la divulgación de la ciencia y la tecnología en todos los niveles de enseñanza.

Cinco años después de la declaración de Caracas, la Organización de Naciones Unidas para la Educación, la Ciencia y la Cultura (en adelante UNESCO) con el apoyo de la Comisión Económica para América Latina y el Caribe (CEPAL) realizaron la primera "Conferencia sobre la Aplicación de la Ciencia y la Tecnología al Desarrollo de América Latina" en Santiago de Chile. En ésta, se consideraron los primeros lineamientos para el desarrollo científico y tecnológico de la región. Según Lemarchand (2010) Esta conferencia fue un hito a nivel mundial porque América Latina fue la primera región de países en vía de desarrollo que planteó la aplicación de la Ciencia y la Tecnología. ${ }^{2}$ p. 89. Lo cual significa un elemento de vanguardia de la región.

Entre los años setenta y ochenta, continuaron las reuniones regionales sobre la aplicación de la ciencia y la tecnología, para determinar cuáles eran las prioridades de ciencia y tecnología en América Latina. Entre las propuestas, se planteó la integración de la ciencia en los sectores productivos de los países miembros de la UNESCO.

Es importante tener presente que la concepción de la ciencia y la tecnología en aquello años era de carácter lineal, ${ }^{3}$ es decir, no tomaba en cuenta elementos económicos o sociales que pudieran afectar el desarrollo de ésta.

Además de la UNESCO, otros organismos internacionales como la Organización de Estado Americanos (en adelante OEA) tomaron interés por la ciencia y la tecnología.

Durante la década de los noventa continuó el proceso de institucionalización del tema. En 1996, fue a probado por los Ministros de Ciencia y Tecnología de los países miembros de la 
OEA la declaración de Cartagena la cual fijó las directrices para la formulación de políticas en materia de ciencia, tecnología e innovación en la región.

En la declaración, los ministros responsables reconocieron varios aspectos, entre ellos la necesidad de; incluir la ciencia y la tecnología en las agendas nacionales para contribuir con el desarrollo socioeconómico y ambiental del hemisferio; formular de políticas para el sector; fortalecer sistemas de innovación orientados al cambio de tecnologías; incluir a todos los sectores de la sociedad (sector privado, comunidad científica, sociedad civil) en la implementación de las políticas; implementar mecanismos de cooperación para el desarrollo científico y tecnológico en los países de la región, entre otros.

Al revisar esta y las anteriores declaraciones, es posible identificar la voluntad politica de las instituciones de cooperación internacional de la región (OEA, UNESCO, CEPAL) en subrayar la importancia de la ciencia, la tecnología y la innovación, así como propiciar la inclusión de los diferentes sectores de la sociedad en la formulación de políticas y generar de mecanismos de cooperación.

Si bien desde 1948 se ha llevado a cabo un proceso de institucionalización de la ciencia, la tecnología y la innovación, todavía es mucho el camino que falta para disminuir la brecha entre éstas y la sociedad, ya que existe una tendencia generalizada en verlas como elementos a los que solo tiene acceso un público selecto: los científicos. Es allí donde cobra importancia la apropiación social de la ciencia, la tecnología y la innovación.

Apropiación Social del conocimiento.

Antes de abordar las leyes, politicas y programas en materia de CTI, conviene precisar el término de apropiación social del conocimiento y el enfoque propuesto por instituciones de cooperación internacional en la región.

La apropiación del conocimiento es un punto de especial interés para los estados miembros de la de OEA, ya que en un documento diagnóstico sobre el tema, se identificó la necesidad de

Incorporar en el sentido común y en la vida cotidiana la lógica de la ciencia como estrategia de interacción con la realidad fisica y social, o en otras palabras, desarrollar una cultura receptiva a la ciencia es entonces un requisito indispensable para el desarrollo de las naciones (Ahumada y Miranda, 2003. p.3).

Por su parte, el Convenio Andrés Bello también ha impulsado el tema de la apropiación social de la ciencia y la tecnología, a través la Política Pública en Apropiación Social de la Ciencia y la Tecnología de los países signatarios de la Organización del Convenio Andrés Bello Entre los propósitos de esta política se encuentran.

...fortalecer los programas y acciones estratégicas regionales y nacionales en este campo, de manera que los ciudadanos y las ciudadanas se interesen, comprendan, innoven, generen y utilicen la ciencia y la tecnología en su vida cotidiana, de acuerdo con las realidades de cada país; permite procesos permanentes de participación y aprovechamiento de los desarrollos científicos y tecnológicos en el mejoramiento de la calidad de vida, y en la solución a problemas cotidianos; crear o fortalecer una cultura científica y tecnológica; y 
mejorar los vínculos entre los diferentes actores y agentes relacionados con la generación y uso del conocimiento científico y tecnológico.(CAB, 2008, p1).

La ciencia la tecnología y la innovación en Colombia.

Al examinar los lineamientos y declaraciones en materia de la Ciencia, la Tecnología y la Innovación (en adelante CTI) para Latinoamérica, es posible entender, las políticas, planes y programas que actualmente cuenta Colombia.

Según la doctora Mónica Lozano (2008), Colombia es uno de los países de la región que ha ejercido un papel fundamental en la discusión política del tema. A finales de los años noventa, el estado Colombiano ha venido adelantando algunos cambios para integrar dentro de la estrategia de desarrollo del país. Dichos cambios se encuentran reflejados en la formulación de leyes, politicas, y planes nacionales de desarrollo.

Ley 1286 de 2009 .

Esta ley propuso cambios de forma y fondo en institucionalización de la CTI, entre ellos es posible puntualizar los siguientes:

- Transformación del Instituto Colombiano para el Desarrollo de la Ciencia y la Tecnología "Francisco José Caldas"-Colciencias-, por el Departamento Administrativo de Ciencia, Tecnología e Innovación-Colciencias- .

- Fortalecimiento del Sistema Nacional de Ciencia, Tecnología, incluyendo el componente de Innovación.

- Consolidación de una política de estado en materia de ciencia, tecnología e innovación.

Para entender mejor estos cambios continuación se describirá cada una de las propuestas:

- Transformación del Instituto Colombiano para el Desarrollo de la Ciencia y la Tecnologia "Francisco José Caldas" -Colciencias-, en el Departamento Administrativo de Ciencia, Tecnología e Innovación-Colciencias- .

Como departamento administrativo, Colciencias adquirió entre sus funciones la de propiciar una cultura basada en "la generación, la apropiación y la divulgación del conocimiento, y la investigación científica, la innovación y el aprendizaje permanente"(Colombia,2009. p.1). Este apartado evidencia la importancia de la apropiación y divulgación social del conocimiento.

A pesar de la transformación del instituto, se conservaron algunos aspectos de la Política Nacional de Fomento a la Investigación y la Innovación de 2008 tales como la adopción del modelo de sistema de investigación e innovación elaborado por Arnold y Kuhlman 2001, como modelo operativo para el sistema Colombiano, lo cual indica una continuidad en las políticas.

Este modelo operativo identifica la interrelación de otros actores que contribuyan al fortalecimiento del sistema de ciencia, tecnología e innovación tal es el caso del sector 
productivo, es decir, ya no es un tema exclusivo de los centros de investigación, la academia o el estado sino que se propone como un tema que incluye a otros actores, como la empresa. Además, tal como lo señala la política de 2008 el modelo también es útil para entender el Sistema Nacional de Ciencia Tecnología e Innovación Colombiano y poder modificar Políticas, incluir actores y fortalecer los flujos de conocimiento e información (Instituto Colombiano, 2008, p. 106).

Es válido preguntarse si este sistema ha contado con el financiamiento necesario por parte del estado Colombiano y de la cooperación internacional.

Al respecto, en 2009, Colciencia gestionó un crédito de 500 millones de dólares al Banco Interamericano de Desarrollo y al Banco Mundial, dicho crédito sería utilizado para la consolidación de capacidades tanto de Colciencias como del Sistema Nacional de Ciencia, Tecnología e Innovación. (Colciencias, 2009).

Los recursos económicos fueron conseguidos, ahora bien, falta conocer los resultados de esa gestión.

- Fortalecer el Sistema Nacional de Ciencia, Tecnología, incluyendo el componente de Innovación.

Para su fortalecimiento, fue necesario transformar el Sistema Nacional de Ciencia y Tecnología, por el Sistema Nacional de Ciencia, Tecnología e Innovación. El cambió tuvo como propósito cumplir lo estipulado en el artículo 16 "con el fin de integrar las actividades científicas, tecnológicas y de innovación bajo un marco donde empresas, Estado y academia interactúen en función de los fines de la presente ley" (Colombia, 1990. P.4).

De igual forma el nuevo sistema promueve y consolidar una visión a largo plazo en materia de CTI, así como generar mecanismos de inversión pública y privada y propiciar la articulación entre los actores en el sistema, en especial la triada-empresa-estado-universidad.

Es de destacar que el tema de innovación es considerada una de las locomotoras del crecimiento económico presente en el Plan Nacional de desarrollo 2010014.

- Consolidar una política de estado en materia de ciencia, tecnología e innovación.

Para fortalecer esta política ley 1286 de 2009, establece que ello será posible si se logra:

Incrementar la capacidad científica, tecnológica, de innovación y competitividad del país para dar valor agregado a los productos y servicios de origen nacional y elevar el bienestar de la población en todas sus dimensiones. (numeral 1, artículo 3, p.1).

Tal como fue indicado en el apartado anterior, el componente de innovación va de la mano con otro componente: la competitividad, que a su vez va alineada dentro la lógica de libre mercado y con los Planes de Desarrollo de Colombia de los últimos años. 
Planes, políticas y estrategias.

A continuación se indicaran planes y politicas que incluyen o la ciencia o la tecnología o la innovación, incluso los tres.

El Plan Nacional de Desarrollo 2010-2014 hace referencia únicamente a la innovación, la cual es vista como uno de los pilares para alcanzar el desarrollo económico sostenible del país. En efecto, el documento resumen del plan la denomina como "un vehículo para alcanzar la prosperidad" (p.8).

En tanto, la Política Nacional de Competitividad y productividad sustentada en el conpes 3527 de 2008, la estrategia Nacional de Apropiación social de la CTI 2010 y en la Estrategia Nacional de Cooperación Internacional 2012-2014 se encuentran presente la ciencia, la tecnología y la innovación.

- Politica Nacional de Competitividad y productividad sustentada en el conpes 3527 de 2008.

Esta política es anterior a la ley 1286, en ella se identifican las bases necesarias para la creación de un sistema de investigación e innovación para que el conocimiento sea un instrumento de desarrollo. Propone seis ejes de trabajo.

El primer eje es el apoyo a la formación para la CTI que abarca no sólo la formación de doctores sino el desarrollo de competencias de niños y jóvenes.

El segundo eje, es la consolidación de capacidades para la CTI, la cual está orientada a fortalecer; la institucionalidad a partir de financiamiento de proyectos, el trabajo en red, la adquisición de bibliografía y equipos especializados.

El tercer eje es la transformación productiva mediante el fomento de la innovación y el desarrollo tecnológico del sector productivo tiene que ver con el impulso del sector empresarial para estar a la altura del mercado global. Plantea "Incrementar los esfuerzos en educación y capacitación que apoyen los procesos de aprendizaje, traducción y apropiación de conocimiento en las empresas" (Colombia, 2008. P.42) lo cual indica la importancia de la diada Universidad Empresa.

El cuarto y quinto eje tienen que ver con la consolidación de la institucionalidad del SNCTIy el fomento a la apropiación social de la CTI en la sociedad colombiana. El primero fue descrito anteriormente y el segundo será abordado en la estrategia de apropiación social de la CTI. Lo importante de ambos ejes es que están articulados con los documentos que lo sustentan.

El sexto y último eje, desarrollo de las dimensiones regional e internacional de la CTI tiene que ver con la descentralización del Sistema Nacional de la CTI y con la internacionalización de los grupos y centros de investigación.

En definitiva, estos ejes dejan en evidencia la tan subrayada necesidad de fortalecer el sistema de la CTI. 


\section{- Politica Nacional de fomento a la Investigación y la Innovación de 2008.}

Al igual que en política recién descrita, en ésta, también valora la relación universidadempresa, la competitividad, la transformación productiva y la cualificación de los investigadores.

De igual forma, subraya la importancia de fortalecer, formular e impulsar políticas para generar una cultura científica a través de la apropiación y la divulgación del conocimiento. Sin embargo, esta politica deja en evidencia el deber ser para el fomento de la CTG pero no indica el cómo se puede concretar los puntos expuestos.

La cultura científica a la que hace mención esta política, está relacionada directamente con la estrategia de apropiación social de la CTI, la cual se describirá a continuación.

- Estrategia Nacional de Apropiación social de la CTI 2010.

Esta estrategia publicada en 2010 es el resultado de los retos propuestos en la ley 1286 de 2009. Como bien señaló el entonces director de Colciencias, Jaime restregó, proporciona las directrices para orientary,

...estimular la creación y consolidación de espacios para la comprensión, reflexión y debate de soluciones a problemas sociales, politicos, culturales y económicos en los cuales la generación y uso de conocimiento científico y tecnológico juegan un papel preponderante... (Colciencias, 2010, p.6).

La estrategia parte de un diagnóstico donde se identificaron algunos vacíos que hacen evidente la separación entre la ciencia y la tecnología con la sociedad. Entre los vacíos se encuentran:

- La ausencia de estrategias y programas concretos de apropiación social de la CTI.

- La escasez de recursos.

- La falta de interés de los expertos.

- El desconocimiento del público en general sobre los códigos y lenguajes de las culturas.

Lo anterior constituye un reto para cada uno de los actores que forman parte del Sistema Nacional de Ciencia, Tecnología e Innovación, entre ellos la academia, quien está llamada a tender puentes entre la ciencia y la sociedad.

Además de los vacíos, la estrategia señala como problema central la asimetría entre los fines y las estrategias de apropiación social, ello se debe a dos supuestos:

- La concepción vertical de la construcción del conocimiento.

- La ambigüedad de la noción de apropiación.

Si bien el diagnostico de estas asimetrías es acertado, la estrategia no plantea la forma como puede ser subsanados. 
En teoría, la estrategia tiene como propósito generar instrumentos que permitan a la innovación y a la investigación como los pilares para el desarrollo económico y social del país. a partir de las siguientes líneas de acción:

1. Participación ciudadana en CTI.

2. Comunicación CTI.

3. Transferencia e Intercambio del conocimiento y;

4. Gestión del conocimiento para la apropiación del conocimiento.

La primera linea, subraya la importancia de la participación ciudadana con los diferentes grupos de interés para la apropiación del conocimiento. Esta relación es el primer paso para que se pueda dar la transferencia y el intercambio de conocimiento señalado en el tercer aspecto.

La segunda línea de acción es la comunicación, la cual es considerada como un elemento de mediación entre la ciencia, la tecnología y la sociedad, apunta a la articulación de actores, siendo el ideal el cambiar el imaginario social que se tiene sobre la ciencia y la tecnología, como elementos ajenos a la cotidianidad para ser visto como "una actividad humana en estrecha relación con nuestro medio, con nuestros problemas y nuestra capacidad de resolverlos" (p.31). Este elemento fue identificado desde la declaración de Caracas de 1960.

Finalmente gestión para la apropiación del conocimiento es la cuarta línea planteada, la cual busca.

"promover procesos de generación y uso de conocimiento más democráticos, responsables y respetuosos de las especificidades culturales y sociales de las comunidades "objeto" o beneficiarias de proyectos de investigación o innovación.” (Colombia, 2010. P. 34).

Sin embargo, la estrategia no señala el cómo se debe realizar los procesos de participación, comunicación, transferencia o gestión del conocimiento así como tampoco señala los mecanismos que pueden ser utilizados para llevarlo a cabo. Esta falencia puede ser vista como un desafio para los actores que conforman el Sistema Nacional de Ciencia, Tecnología e Innovación, así como una provocación para abrir un espacio de discusión entre Colciencias y los diferentes actores.

\section{- Estrategia Nacional de Cooperación Internacional 2012-2014. ${ }^{4}$}

Colombia cuenta una destacada trayectoria que en materia de Cooperación internacional, más de cuarenta años de institucionalidad le otorga un puesto destacado en la región en esta materia. La estrategia 2012-2014, reconoce que el país pasó de ser un país de renta media lo cual la hacer no solo demandante sino también oferente de cooperación.

Entre las áreas de demanda de cooperación, se encuentra el crecimiento económico y competitividad, que es uno de los componentes donde se buscar el apoyo de la cooperación internacional en materia de ciencia y tecnología.

Dicho apoyo se refleja a partir del plan de cooperación para incentivar alianzas entre grupos de investigación y con ello favorecer la transferencia de conocimiento y tecnología a partir de la realización de proyectos en conjunto. (Colombia, 2012). Ello se debe a que el conocimiento 
y la innovación son uno de los elementos transversales de las locomotoras del Plan Nacional de Desarrollo.

Es interesante encontrar que entre las líneas prioritarias de cooperación se encuentre el fomento a la apropiación social de la CTI en la sociedad colombiana. Lo que indica que es, además de ser un tema de interés en la estrategia nacional de cooperación internacional, está alineado con los planes y estrategias anteriormente señalados. Pero al igual que los otros, presenta la misma falencia, tampoco plantea el cómo.

La consecución de recursos para financiar la CTI es un punto que resulta álgido debido a que para ver los frutos de la CTI se requiere de tiempo, y por lo general los financiadores les interesa revisar los resultados en corto o mediano plazo. 


\section{Referencias.}

Politica publica en apropiacion social de la ciencia y la tecnologia de los paises signatorios de la organizacion del convenio Andres Bello. (2008). Obtenido de http://convenioandresbello.org/documentos_cyt/POLITICA_PUBLICA_ASCyT_CAB .pdf.

Plan Nacional de desarrollo 2010-214 "prosperidad para todos". (2010). Recuperado el 10 de Diciembre de 2012, de https://www.dnp.gov.co/LinkClick.aspx?fileticket=4-J9VFE2pI\%3d\&tabid=1238.

Ahumada, J., M. F. (2003). Ciencia, tecnologia y sociedad: Algunas reflexiones Documento para la organizacion de Estados americanos. Recuperado el 18 de Noviembre de 2012, de http://www.science.oas.org/doc/policy/ahumada_cyt03_26_04.pdf.

Colciencias. (2009). Firmada primera fase del credito Colciencias con la Banca Multilateral. Recuperado el 10 de Diciembre de 2012, de http:/ / www.colciencias.gov.co/noticias / firmada-primera-fase-del-cr-ditocolciencias-con-la-banca-multilateral.

Estrategia Nacional de Apropiacion Social de la Ciencia, la Tecnologia y la Innovacion. (2010). Recuperado el 23 de Julio de 2012, de http://aprendeenlinea.udea.edu.co/1ms/moodle/file.php/635/ESTRATEGIA_NACI ONAL_DE_ASCTI.pdf.

Estrategia Nacional de Cooperacion Internacional 2012-2014. (s.f.).

Instituto Colombiano para el dasarrollo de la ciencia y la tecnologia, Francisco Jose De Caldas. (2008). Colciencia Consejo Nacional De Ciencia y Tecnologia.

Lamarchand. (2010). Sistema nacionales de ciencia, tecnologia e innovacion en America Latina y el Caribe. Recuperado el 23 de Noviembre de 2012, de http:/ / www.unesco.org.uy/ci/fileadmin/ciencias\%20naturales/Politicas\%20Cienti ficas/EYDPCALC-Vol-1.pdf.

Ley 1286 de 2009, (23 de enero) Diario Oficial, 47241. (s.f.). Recuperado el 20 de Julio de 2012, de

http://www.colciencias.gov.co/sites/default/files/upload/reglamentacion/ley_128 6_de_2009.pdf.

Lozano, M. (2008). El nuevo contrato social sobre la ciencia retos para la comunicacion de la ciencia en America Latina Razon y palabra $N^{\circ}$. 65. Recuperado el 10 de Diciembre de 2012, de http:/ / www.razonypalabra.org.mx/rypant/N/n65/actual/mlozano.html.

Politica Nacional de Competitividad y Productividad. (2008). Recuperado el 20 de Noviembre de 2012, de http://wsp.presidencia.gov.co/sncei/politica/Documents/Conpes3527-23jun2008.pdf.

UNESCO. (1999). Declaracion sobre la ciencia y el uso del saber cientifico. Recuperado el 10 de Diciembre de 2012, de

http://www.unesco.org/science/wcs/esp/declaracion_s.htm\#sociedad. 
${ }^{2}$ En 1963 se realizó en Ginebra otra conferencia similar pero enfocada en beneficio de países menos desarrollados y vista desde los países industrializados.

${ }^{3}$ Monica Lozano (2008) define el modelo lineal de la ciencia como :

el proceso de ascender desde el atraso al desarrollo -o desde la tradición a la modernidad- ya sea subiendo los peldaños de una escalera, en lo esencial única y ya conocida, o ya sea siguiendo una linea recta que avanza inexorablemente.

${ }^{4}$ Dicha estrategia se encuentra en línea con el Plan Nacional de Desarrollo "Prosperidad para Todos" y otros instrumentos como el Conpes 3582. 